\title{
HIV Disease Progression in the First Year After Delivery Among African Women Followed in the HPTN 046 Clinical Trial
}

\author{
D. Heather Watts, MD, * Elizabeth R. Brown, ScD, $\uparrow 99$ Yvonne Maldonado, MD, $\neq$ Casey Herron, MS, $† 99$ \\ Tsungai Chipato, MD, $\S$ Leanne Reddy, MS, || Dhayendre Moodley, MD, || Clemensia Nakabiito, MD, $\mid$ \\ Karim Manji, MD,\# Wafaie Fawzi, MD, ** Kathleen George, MPH, $+\dagger$ Paul Richardson, MS, $\neq$

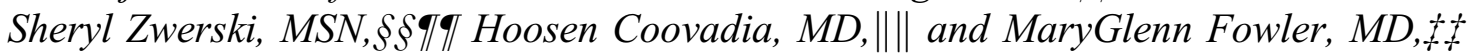 \\ for the HPTN 046 Protocol Team
}

Background: Starting lifelong antiretroviral therapy (ART) in HIVinfected pregnant women may decrease HIV progression and transmission, but adherence after delivery may be difficult, especially for

Received for publication February 28, 2013; accepted June 12, 2013

From the *Maternal and Pediatric Infectious Disease Branch, Eunice Kennedy Shriver National Institute of Child Health and Human Development, NIH, Bethesda, MD; †Fred Hutchinson Cancer Research Center, Seattle, WA; †epartment of Pediatrics, Stanford University School of Medicine, Palo Alto, CA; §University of Zimbabwe College of Medicine, Harare, Zimbabwe; $\|$ Centre for the AIDS Programme of Research in South Africa (CAPRISA), Nelson R Mandela School of Medicine, University of KwaZulu Natal, Durban, South Africa; $\uparrow$ Makerere University-Johns Hopkins University Research Collaboration, Kampala, Uganda; \#Muhimbili University of Health and Allied Sciences, Dar es Salaam, Tanzania; **Harvard School of Public Health, Boston, MA; †Family Health International, Research Triangle Park, Chapel Hill, NC; ††Johns Hopkins Medical Institutes, Baltimore, MD; $\S \S$ National Institute of Allergy and Infectious Diseases, NIH, Bethesda, MD; ||||Maternal Adolescent and Child Health, University of the Witwatersrand, Johannesburg, South Africa, and $\uparrow \uparrow$ Statistical Center for HIV/AIDS Research and Prevention (SCHARP), Seattle, WA

This study was supported by the HIV Prevention Trials Network (HPTN) and sponsored by the National Institute of Allergy and Infectious Diseases, National Institute of Child Health and Human Development, National Institute on Drug Abuse, National Institute of Mental Health, and Office of AIDS Research, of the National Institutes of Health, US Department of Health and Human Services under award U01 AI046749. Overall support for the International Maternal Pediatric Adolescent AIDS Clinical Trials Group (IMPAACT) was provided by the National Institute of Allergy and Infectious Diseases (NIAID) (U01 AI068632), the Eunice Kennedy Shriver National Institute of Child Health and Human Development (NICHD), and the National Institute of Mental Health (NIMH) (AI068632). The content is solely the responsibility of the authors and does not necessarily represent the official views of the NIH. Support of the sites was provided by the National Institute of Allergy and Infectious Diseases (NIAID) and the NICHD International and Domestic Pediatric and Maternal HIV Clinical Trials Network funded by NICHD (contract number N01-DK-9-001/HHSN267200800001C). The study products were provided for free by Boehringer-Ingelheim.

The authors have no conflicts of interest to disclose.

Supplemental digital content is available for this article. Direct URL citations appear in the printed text and are provided in the HTML and PDF versions of this article on the journal's Web site (www.jaids.com).

Correspondence to: D. Heather Watts, MD, Maternal and Pediatric Infectious Disease Branch, Eunice Kennedy Shriver National Institute of Child Health and Human Development, 6100 Executive Blvd, Room 4B11, MSC 7510, Bethesda, MD 20892-7510 (e-mail: wattsh@mail.nih.gov).

Copyright $(2013$ by Lippincott Williams \& Wilkins asymptomatic women. We evaluated disease progression among HIVinfected women not on ART with $\mathrm{CD}^{+}$lymphocyte counts above 200 cells per microliter at delivery.

Methods: We analyzed risk of death, progression to AIDS (stage IV or CD4 $<200$ cells per microliter), or to $\mathrm{CD}^{+}$count $<350$ 1 year after delivery among postpartum women enrolled to a prevention of breastfeeding transmission trial using the Kaplan-Meier method. In the primary analysis, women were censored if ART was initiated.

Results: Among 1285 women who were not WHO stage IV or less at 6 weeks postpartum, $49(4.3 \%)$ progressed to stage IV/CD4 $<200$ cells per microliter or death by 1 year. Progression to CD $4<200$ cells per microliter or death occurred among $16(4.3 \%)$ of 441 women with CD4 count of 350-549 cells per microliter and $10(1.6 \%)$ of 713 with CD4 counts $>550$ cells per microliter at delivery. CD4 $<350$ cells per microliter by 12 months postpartum occurred among $116(37.0 \%)$ of 350 women with CD4 count 400-549 cells per microliter and 48 (7.4\%) of 713 with CD4 count $>550$ cells per microliter at delivery.

Conclusions: Progression to AIDS or CD4 count $<350$ cells per microliter is uncommon through 1 year postpartum for women with CD4 counts over 550 cells per microliter at delivery, but occurred in over one third of those with CD4 counts under 550 cells per microliter. ART should be continued after delivery or breastfeeding among women with CD4 counts $<550$ cells per microliter if follow-up and antiretroviral adherence can be maintained.

Key Words: HIV, postpartum, disease progression

(J Acquir Immune Defic Syndr 2013;64:299-306)

\section{BACKGROUND}

A recent World Health Organization (WHO) programmatic update has suggested that all HIV-infected pregnant women should be considered for initiation of triple antiretroviral (ARV) regimens for prevention of mother-to-child transmission with consideration given to continuing all women on ARVs for life once initiated, regardless of starting $\mathrm{CD}^{+}{ }^{+}$lymphocyte count. ${ }^{1}$ The recommendation for use of triple ARV regimens, rather than zidovudine alone during 
pregnancy with additional peripartum ARV drugs for women with high $\mathrm{CD}^{+}$cell counts, is based on several considerations. Rapid initiation of combination ARV drugs without waiting for $\mathrm{CD} 4$ results in settings where $\mathrm{CD}^{+}$cell counts are often delayed assures treatment for women with low $\mathrm{CD}^{+}$lymphocyte counts who have the highest risk of transmission. ${ }^{2}$ Using the same regimen for all pregnant women and other adults allows for streamlining of drug supply and provider training. In addition, treating HIV-infected persons at higher $\mathrm{CD}^{+}$cell counts resulted in lower risk of transmission to sexual partners in a recent trial. ${ }^{3}$

However, the risks versus benefits of continuing triple antiretroviral regimens started among generally healthy asymptomatic pregnant women with higher $\mathrm{CD}^{+}$lymphocyte counts after delivery or cessation of breastfeeding have not been evaluated adequately. The postpartum period is known in both resource-rich and resource-poor settings to be a high risk period for poor HIV treatment adherence, given the demands of newborn care and frequent lack of disclosure with recent diagnosis during pregnancy. ${ }^{4}$ Especially in resource-limited settings, there is increased potential for disruption in care as traditionally many women travel away from home after delivery for extended stays with family, frequently far from ARV treatment centers. In addition, resource constraints at the country level may preclude provision of longterm therapy for women with higher $\mathrm{CD}^{+}$lymphocyte counts after cessation of breastfeeding without sacrificing treatment access for those who already meet WHO and the Ministry of Health treatment criteria.

Rates of disease progression among asymptomatic $\mathrm{HIV}$-infected women during the first year after delivery have not been well documented in resource-limited settings. Thus, the purpose of this analysis was to assess HIV immunologic and clinical disease progression during the first 12 months postpartum among asymptomatic HIV-infected women with higher CD4 counts at delivery using data from a recently completed trial, HIV Prevention Trials Network (HPTN) 046.

\section{METHODS}

HPTN 046 was a randomized, double-blinded placebocontrolled trial of an extended nevirapine regimen in infants for preventing the transmission of HIV through breastfeeding conducted in Zimbabwe, South Africa, Uganda, and Tanzania. ${ }^{5}$ The protocol was approved by all relevant institutional review boards and regulatory bodies; all women provided written consent for themselves and their infants before enrollment.

This analysis includes women who provided informed consent and were screened during the third trimester of pregnancy up to 7 days postpartum whose infants were eligible and randomized. To be eligible, women had to have confirmed HIV-1 infection, be at least 18 years old, intend to breastfeed, and not have any serious medical condition that would interfere with breastfeeding or study participation. Their infants had to be HIV uninfected at 3 days of life under version 2.0 and at 6 weeks of age in version 3.0. Maternal care and follow-up were the same in both versions. Maternal ARV regimens were not provided through the study but were available in accordance with the local standard of care, which primarily consisted of combination ARV therapy for women with $\mathrm{CD}^{+}$lymphocyte counts below 200 cells per microliter and maternal and infant single-dose nevirapine for those with higher $\mathrm{CD}^{+}$cell counts. Enrollment occurred between February 2007 and March 2010. Infants were enrolled at 3 days postpartum under version 2.0 and at 6 weeks postpartum in version 3.0, but all women were enrolled by 7 days postpartum and had study visits at delivery or within 7 days postpartum, at 2 and 6 weeks, and at 3, 6, and 12 months postpartum. At each visit, an interim medical history and symptom-directed physical examination were completed. Maternal HIV disease staging was completed using the WHO staging system, ${ }^{6}$ and a complete blood count and $\mathrm{CD}^{+}$lymphocyte count were obtained in laboratories certified by the National Institute of Allergy and Infectious Diseases (NIAID) Division of AIDS Quality Assurance Program (Figs. 1 and 2)

\section{Statistical Analyses}

To assess maternal disease progression over time, we used the Kaplan-Meier method to estimate the cumulative proportion of mothers who progressed to 1 of 4 surrogate disease end points at 6 and 12 months postpartum. Two of the end points described $\mathrm{CD} 4^{+}$lymphocyte decline from baseline at delivery: time to (1) $\mathrm{CD}^{+}$lymphocyte counts below 200 cells per microliter and (2) $\mathrm{CD}^{+}$lymphocyte counts below 350 cells per microliter. Women were censored at the time of ART initiation. The other 2 end points described progression to clinical AIDS or death from 6 weeks postpartum: (3) WHO clinical stage III or IV or death and a combined end point of (4) WHO clinical stage IV, CD4 ${ }^{+}$lymphocyte count below 200 cells per microliter, or death. Mothers were excluded from the analysis of the first 2 end points if they had delivery $\mathrm{CD}^{+}$lymphocyte counts below 200 and 350 cells per microliter, respectively, whereas the third analysis excluded those mothers who were stage III or IV at 6 weeks postpartum or who had started ART before 6 weeks; and finally, the fourth analysis excluded those mothers who were stage IV at 6 weeks, had taken ART before six weeks, or had $\mathrm{CD}^{+}$lymphocyte counts below 200 cells per microliter before 6 weeks. For the combined end points, 3 and 4, time to event was calculated as the minimum event time if a mother had more than one of the qualifying outcomes. For the third end point, women were censored at time of ARV initiation after 6 weeks or at the last visit through 12 months postpartum if they did not die or progress to WHO stage III or IV. For the fourth end point, women were censored at the time of ARV initiation after 6 weeks postpartum or at last $\mathrm{CD}^{+}$measure above the threshold of 200 cells per microliter through 12 months postpartum if they did not die or progress to WHO stage IV. Because ARV initiation is likely to be informative, a combined end point analysis was also performed where the time to event was the minimum visit in which the above thresholds (points 1-4) were crossed or ARV treatment was initiated. All participants in whom the end point was not observed were censored at their last visit time. Maternal death incidence was calculated by dividing the number of deaths by the total person-years through 12 months of follow-up and stratified by $\mathrm{CD}^{+}$lymphocyte counts at delivery. 

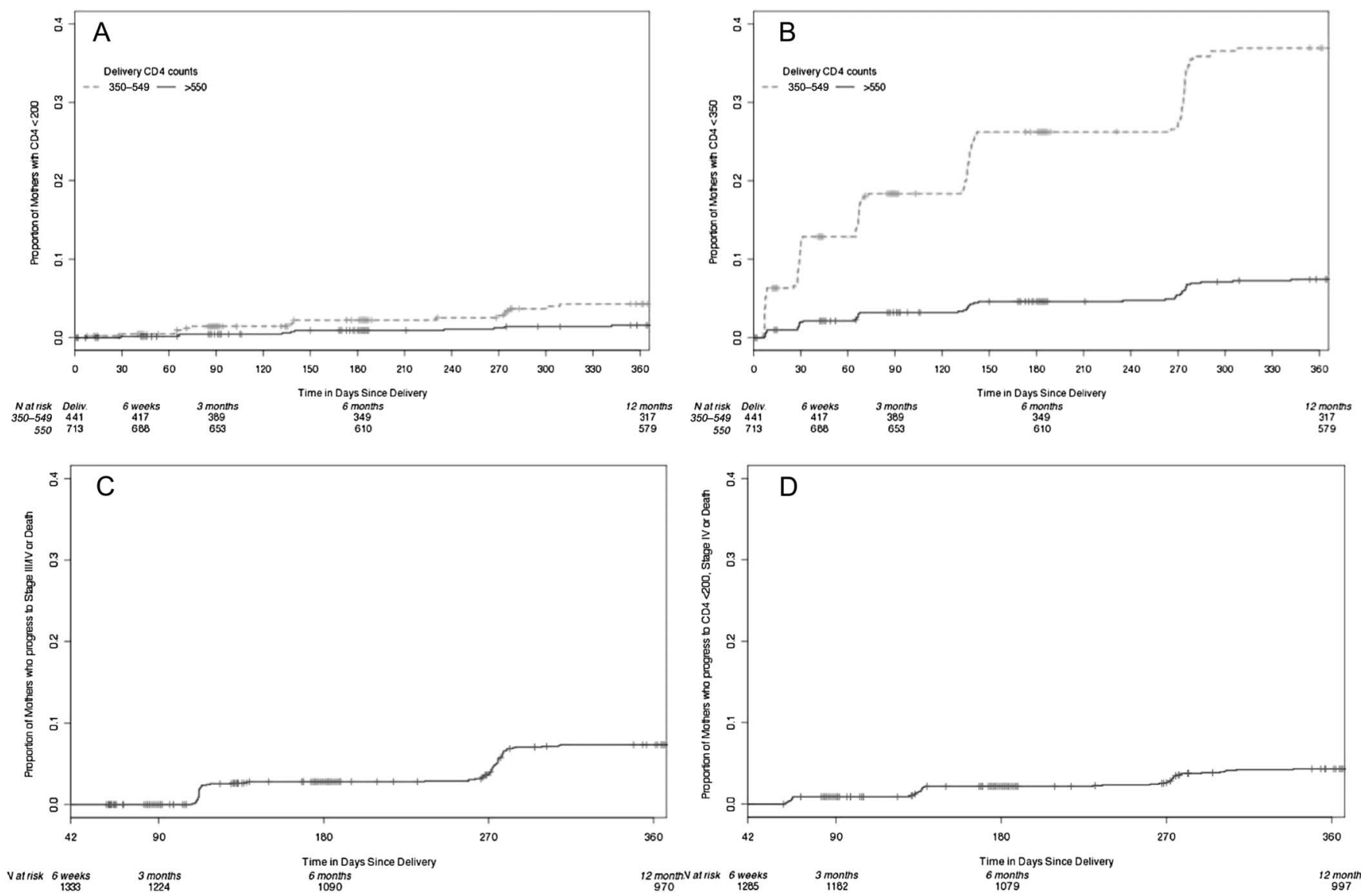

FIGURE 1. Time to (A) CD4+ lymphocyte counts below 200 cells per microliter, (B) CD4+ lymphocyte counts below 350 cells per microliter, (C) WHO clinical stage III/IV or death, and (D) WHO clinical stage IV, CD4+ count $<200$ cells per microliter, or death.

\section{RESULTS}

From February 2007 to March 2010, 2025 women enrolled into HPTN 046, including 1430 (70.7\%) with CD4 ${ }^{+}$ lymphocyte counts above 350 cells per microliter (Table 1). WHO clinical staging was available at 6 weeks postpartum in 1945 women; $1855(96 \%)$ were stage I or II. Women without WHO clinical staging at 6 weeks had similar demographic characteristics and baseline $\mathrm{CD}^{+}$lymphocyte counts compared with those with staging (data not shown). Twenty-eight percent of the women received triple ART regimens during pregnancy. Other baseline characteristics are summarized in Table 1.

Maternal disease progression rates both with censoring at ARV initiation and with ARV initiation included in the end point are summarized in Table 2. Among women who had $\mathrm{CD}^{+}$cell count results available at delivery and postpartum sampling (Table 2), 16 (4.3\%) of those with counts between 350 and 549 cells per microliter dropped to below 200 cells per microliter by 1 year compared with $10(1.6 \%)$ of those starting above 549 cells per microliter (Fig. 1A). Among women who had a $\mathrm{CD} 4^{+}$lymphocyte count between 400 and 549 cells per microliter at delivery, $116(37 \%)$ dropped under 350 cells per microliter by 1 year after delivery, whereas 48 (7.4\%) of those with a $\mathrm{CD} 4^{+}$cell count of $\geq 550$ cells per microliter at delivery dropped below this threshold (Fig. 1B). When adding ARV initiation to the end point rather than censoring, rates of the end point of $\mathrm{CD}^{+}$count $<200$ cells per microliter or ARV initiation more than doubled for women with $\mathrm{CD} 4^{+}$counts between 350 and 549 cells per microliter but did not change appreciably for the end point including dropping to $\mathrm{CD}^{+}$count below 350 cells per microliter or for those starting above a $\mathrm{CD}^{+}$ count of 550 cells per microliter (Figure 2). For women who started in stages I to III, 49 (4.3\%) died or progressed to AIDS based on stage IV conditions or $\mathrm{CD} 4^{+}$lymphocyte count below 200 cells per microliter by 12 months after delivery, censoring for ARV initiation (Fig. 1C and D; Table 2). When including ARV initiation in the end point, 88 (7.7\%) met the progression criteria. By 12 months, 83 (7.4\%) women starting in clinical stage I or II progressed to stage III or IV, whereas 159 (13.4\%) progressed to stage III or IV or initiated ARV. Of the 74 women initiating ARV by 12 months after delivery, 57 (77\%) had nadir $\mathrm{CD}^{+}$lymphocyte counts below 200 cells per microliter before initiation, $10(14 \%)$ were between 200 and 300 cells per microliter, and $7(9 \%)$ remained above 300 cells per microliter. The reasons for initiation of ARV at higher $\mathrm{CD} 4^{+}$counts were not documented.

Twelve (1\%) women who were not stage IV at their 6-week postpartum assessment developed 13 clinical stage IV end points by 12 months after delivery. These end points included 5 cases of extrapulmonary tuberculosis, 2 cases each of HIV wasting ( $>10 \%$ weight loss plus either unexplained 

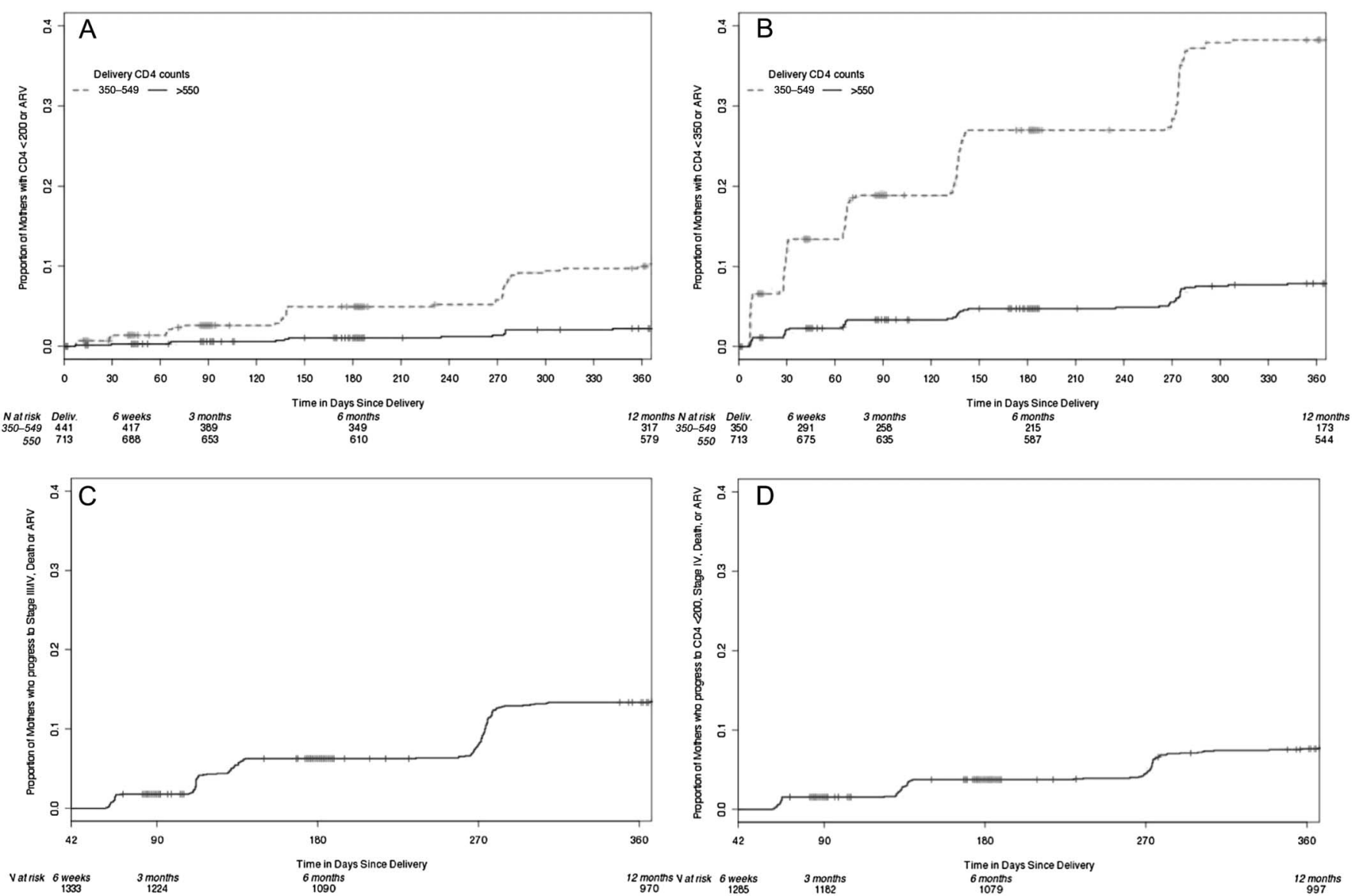

FIGURE 2. Time to ART initiation or (A) CD4+ lymphocyte counts below 200 cells per microliter, (B) CD4+ lymphocyte counts below 350 cells per microliter, (C) WHO clinical stage III/IV or death, and (D) WHO clinical stage IV, CD4 ${ }^{+}$count $<200$ cells per microliter, or death.

diarrhea for over 30 days or chronic weakness and unexplained fever for over 30 days), Pneumocystis jiroveci pneumonia, and extrapulmonary cryptococcosis, and 1 case each of central nervous system toxoplasmosis and symptomatic HIV-associated nephropathy/cardiomyopathy. Sixteen $(0.8 \%)$ of enrolled women died during the first year after delivery (see Table S1, Supplemental Digital Content, http://links.lww.com/QAI/A443). Cause of death was unknown in 7 cases and had varied causes in the other 9 . Only 1 of the 9 deaths (11\%) with known causes was potentially secondary to an AIDSdefining condition and only 2 women who died had a $\mathrm{CD}^{+}$cell count below 200 cells per microliter at the visit before death. Maternal death rates per 100 person-years stratified by $\mathrm{CD}^{+}$lymphocyte count at delivery are shown in Table 3 . The rates of death did not differ significantly by CD4 stratum, but number of events were low. Eleven $(68.8 \%)$ of the deaths occurred among women with $\mathrm{CD} 4^{+}$ lymphocyte counts over 350 cells per microliter at the visit before their death.

\section{DISCUSSION}

The pregnant and postpartum HIV-infected women enrolled to this trial were representative of the women seen at clinical research sites in sub-Saharan Africa ${ }^{7,8}$ and had a range of $\mathrm{CD}^{+}$lymphocyte counts at delivery, with $70 \%$ above 350 cells per microliter, the current threshold for initiation of ARV therapy in many countries. ${ }^{9}$ Less than $5 \%$ had stage III or IV disease at baseline, whereas $9 \%$ had $\mathrm{CD} 4^{+}$ lymphocyte counts below 200 cells per microliter, suggesting that symptoms do not reliably indicate those most in need of ARV therapy. In addition, with the current $\mathrm{CD}^{+}$count threshold of 350 cells per microliter, symptoms will not identify many of those who need therapy.

Given the standard of care existing at the sites during the HPTN 046 trial, asymptomatic women with $\mathrm{CD}^{+}$lymphocyte counts above 350 cells per microliter did not meet WHO or country-specific criteria for ARV therapy. Within this context, we carefully monitored the women study participants for disease progression to initiate treatment if needed. This close follow-up also provided the opportunity to help inform future treatment recommendations. Women who were asymptomatic (WHO clinical stage I or II at baseline) had a relatively low risk $(7.4 \%)$ of progressing to symptomatic HIV disease within 1 year postpartum, emphasizing the need for $\mathrm{CD}^{+}$lymphocyte testing to target therapy for those individuals at highest risk for disease progression in settings where universal treatment is unaffordable. Among women 
TABLE 1. Maternal Baseline Characteristics $(n=1995)$

\begin{tabular}{lc}
\hline Characteristic & N (\%) \\
\hline Enrollment site & \\
Chitungwiza, Zimbabwe & 664 \\
Kampala, Uganda & 705 \\
Dar es Salaam, Tanzania & 218 \\
Durban, South Africa & 408 \\
Median Age, yr (range) & $27(18-46)$ \\
Marital status & \\
Never married/not living with partner & $596(29 \%)$ \\
Married & $757(37 \%)$ \\
Living with partner & $604(30 \%)$ \\
Separated/divorced/widowed & $68(3 \%)$ \\
Antiretrovirals received during pregnancy before delivery & - \\
None or only intrapartum prophylaxis & $583(25 \%)$ \\
1 or 2 drugs & $868(43 \%)$ \\
3 drugs & $574(28 \%)$ \\
CD4 $4^{+}$lymphocyte count (cells/ $\mu$ L; $\left.n=2022\right)$ & - \\
$<200$ & $171(9 \%)$ \\
$200-349$ & $421(21 \%)$ \\
$350-549$ & $594(29 \%)$ \\
$\geq 550$ & $836(41 \%)$ \\
WHO stage assessed at 6 wk postpartum $(\mathrm{n}=1945)$ & - \\
I & $1568(81 \%)$ \\
II & $287(15 \%)$ \\
III & $84(4 \%)$ \\
IV & $6(<1 \%)$ \\
\hline
\end{tabular}

with $\mathrm{CD}^{+}$cell counts between 400 and 549 cells per microliter at baseline, 37\% dropped to below 350 cells per microliter at 1 year, indicating a need for ARV therapy and suggesting that women in this range should be offered continuation of therapy after cessation of perinatal transmission risk. These findings are similar to data from the multicountry MTCT-Plus Initiative, which found that women stopping a variety of ARV regimens for prevention of perinatal transmission had a $46 \%$ risk of dropping below 350 cells per microliter by 24 months postpartum when the initial $\mathrm{CD}^{+}$ cell count during pregnancy was 400-499 cells per microliter. ${ }^{10}$ A study from Haiti found that women stopping antiretroviral prophylaxis at delivery with a $\mathrm{CD}^{+}$lymphocyte count between 350 and 499 cells per microliter dropped to the threshold of 350 cells per microliter requiring therapy at a median of 19 months after delivery compared with a median of 71 months to reach this threshold among women with $\mathrm{CD}^{+}$cell counts at or above 500 cells per microliter at delivery. ${ }^{11}$ These data are also consistent with a study from Brazil, which showed that among women discontinuing ARV agents after delivery, the group with levels between 250 and 500 cells per microliter had a risk of progression to stage II or III events that was 2.5 times higher than women with $\mathrm{CD}^{+}$counts above 500 cells per microliter. ${ }^{12}$ The importance of $\mathrm{CD}^{+}$ lymphocyte results for predicting progression were also shown in a study from Kenya demonstrating that CD4 counts and percentage during pregnancy were most predictive of mortality over the first 2 years postpartum compared with total lymphocyte count, hemoglobin, HIV RNA level, or body mass index. ${ }^{8}$

Among women in the 350-550 cells per microliter $\mathrm{CD}^{+}$lymphocyte group, continuation would also have benefits in reduction of heterosexual transmission to discordant partners. This conclusion is based on the results from a large randomized trial of discordant couples, which found that treating HIV-infected subjects with $\mathrm{CD} 4^{+}$lymphocyte counts between 350 and 550 cells per microliter reduced linked transmissions to partners by $96 \%$ as compared with delaying therapy until the $\mathrm{CD}^{+}$lymphocyte count drops below 250 cells per microliter or symptomatic illness occurred. ${ }^{3}$ Although earlier treatment at higher $\mathrm{CD}^{+}$levels above 550 cells per microliter may also reduce the risk of sexual transmission, the benefit in the group with higher counts has not been proven. Women beginning ARVs in pregnancy with $\mathrm{CD}^{+}$cell counts between 350 and 550 cells per microliter should be counseled about the potential benefit of reduced sexual transmission when they are considering whether or not to continue ARVs after delivery.

Intensive counseling for adherence to the regimen must be provided for women who choose to continue ARVs after cessation of mother-to-child HIV transmission risk, as adherence has been shown to decrease markedly once the incentive of transmission prevention has ended. A recent meta-analysis reported a pooled estimate of adequate adherence $(>80 \%$ of doses) of $75.7 \%$ [95\% confidence interval (CI): $71.5 \%$ to $79.7 \%$ ] during pregnancy compared with $53.0 \%$ (95\% CI: $32.8 \%$ to $72.7 \%, P=0.005)$ during the postpartum period. ${ }^{4}$ This study included women from a range of low-, middle-, and high-income countries. Likewise, in several reports from African sites, HIV-infected women were at particularly high risk for loss to follow-up with $\geq 50 \%$ defaulting on their maternal child health appointments after delivery in some studies. ${ }^{13-16}$ Travel away from home to stay with family for several months after the birth of a child is common. In addition, many women have not disclosed their HIV status to partners or family members making adherence challenging, especially if residing with relatives. ${ }^{17}$ Poor adherence increases the risk of development of viral resistance and treatment failure. ${ }^{18}$ Concerns regarding development of resistance may be especially important, given the current WHO recommendations for efavirenz-based firstline therapies that require a single mutation for resistance. ${ }^{9}$ Clearly, support for adherence and follow-up after delivery will need to be intensified for women planning to continue ARV agents postpartum.

In our study, women with $\mathrm{CD}^{+}$lymphocyte counts of 550 cells per microliter or higher had a lower risk of disease progression with only $7.4 \%$ dropping below 350 cells per microliter and $1.6 \%$ below 200 cells per microliter by 1 year postpartum without ARVs. These results are consistent with data from the MTCT-Plus Initiative, which demonstrated that $18.5 \%$ of women with initial CD4 ${ }^{+}$cell counts above 500 cells per microliter during pregnancy progressed to counts below 350 cells per microliter by 24 months after delivery. ${ }^{10}$ The group of women with $\mathrm{CD}^{+}$cell counts above 550 cells per microliter would be expected to have a low risk of disease progression even with discontinuation of ARVs after pregnancy and breastfeeding, so could discontinue therapy if 
TABLE 2. Cumulative Rates of (A) CD4 + Cell Decline or Death Stratified by CD4+ Cell Count at Delivery and (B) Progression to Clinical AIDS or Death at 6 and 12 months Among Women Not on Combination Antiretroviral Therapy Before Delivery

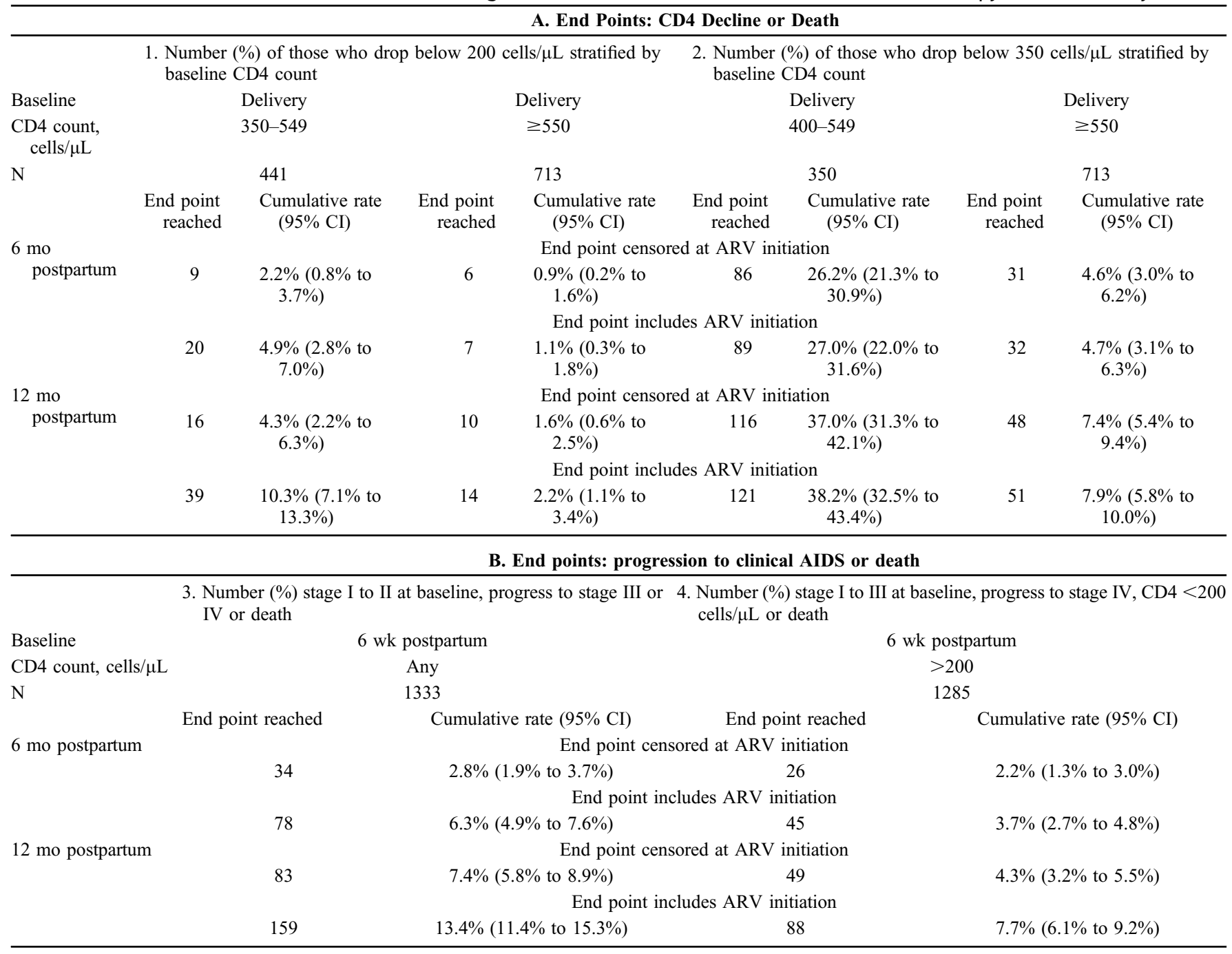

desired. These findings underscore the utility of $\mathrm{CD}^{+}$lymphocyte testing and suggest that in this group of women, more data are needed regarding the risks and benefits of continuing ARVs after risk of mother-to-child transmission of HIV has passed.

There is also a need for operational research that investigates better ways to achieve high levels of adherence to ARV drugs among both pregnant and postpartum women

TABLE 3. Maternal Death Rates per 100 Person-years Stratified by CD4+ Lymphocyte Count

\begin{tabular}{lcccc}
\hline $\begin{array}{l}\text { CD4 at } \\
\text { Delivery }\end{array}$ & Deaths & Person-years & $\begin{array}{c}\text { Death Rate (Deaths } \\
\text { per } \mathbf{1 0 0} \text { person-years) }\end{array}$ & 95\% CI \\
\hline$<200$ & 2 & 132.47 & 1.51 & 0.18 to 5.45 \\
$200-349$ & 2 & 353.08 & 0.57 & 0.07 to 2.05 \\
$350-550$ & 5 & 510.06 & 0.98 & 0.32 to 2.29 \\
$>550$ & 7 & 726.90 & 0.96 & 0.39 to 1.98 \\
\hline
\end{tabular}

304 | www.jaids.com and to decrease loss to follow-up among HIV-infected pregnant women. In areas where implementation of universal treatment is currently not feasible for cost, supply, and logistic reasons, targeting therapy to groups at highest risk for disease progression and transmission to their infants based on their $\mathrm{CD}^{+}$ cell counts remains necessary. Even with implementation of the strategy of starting lifelong therapy for all pregnant women, $\mathrm{CD}^{+}$lymphocyte testing may be helpful to target women with low $\mathrm{CD}^{+}$cell counts for increased support and provision of opportunistic infection prophylaxis. Events occurring among the postpartum women were rare but similar to those reported in the HPTN052 study discussed above, with deaths $(0.8 \%)$ and extrapulmonary tuberculosis $(0.2 \%)$ being the most frequent serious events in the current study. ${ }^{3}$ Causes of death were heterogeneous with only 2 cases occurring with proximate $\mathrm{CD}^{+}$lymphocyte counts under 200 cells per microliter and only 1 case (probable meningitis) likely AIDS defining. Other infections (malaria and hepatitis) and comorbidities (anemia, 
diabetes, pancreatitis, and cardiac failure) contributed to deaths, and some of these conditions may have been ameliorated by earlier ARV. Incomplete data on causes of death limit the conclusions that can be drawn.

Availability of ARV therapy for women with $\mathrm{CD} 4^{+}$ lymphocyte counts below 200 cells per microliter in the current HPTN 046 study seems to have lowered the risk of death compared with earlier studies in similar populations. The HPTN 024 study, conducted among pregnant women in Malawi, Zambia, and Tanzania between 2001 and 2003, at a time when antiretroviral therapy was not widely available in those settings, reported death rates over the first year postpartum of 6.1 per 100 person-years among women with $\mathrm{CD} 4^{+}$ lymphocyte counts below 200 cells per microliter at delivery, 1.1 per 100 person-years with $\mathrm{CD}^{+}$cell count of $200-500$ cells per microliter, and 0.4 per 100 person-years for those with $\mathrm{CD}^{+}$cell counts over 500 cells per microliter. No deaths occurred among 331 HIV-uninfected women in the study. ${ }^{19}$ No ARV therapy was available to women after delivery in that study. In a retrospective review of a cohort of women delivering in Malawi in 2008 who received postpartum ARV therapy if they had WHO stage III or IV disease or $\mathrm{CD} 4^{+}$lymphocyte count below 250 cells per microliter, the mortality rate over 18-20 months postpartum was 4.24 deaths per 100 personyears among 173 consecutive HIV-infected women and 0 among HIV-uninfected delivering at the same sites. ${ }^{20}$

There are certain limitations to these findings including incomplete data on causes of death and lack of longer term follow-up. Data on the use of hormonal contraception are not available, but unlikely to have influenced the findings, based on data from Kenya among postpartum women that found no differences in $\mathrm{CD}^{+}$cell count or HIV RNA changes with use of hormonal contraceptives compared with no use. ${ }^{21}$ Despite these limitations, there are also a number of strengths of the HPTN 046 study including the large number of participants followed in 4 African countries and excellent retention that allowed careful monitoring of serial $\mathrm{CD} 4{ }^{+}$lymphocyte counts and end point ascertainment over the first year after delivery. Because WHO and in-country guidelines in place during the study did not recommend ARV treatment for asymptomatic individuals with $\mathrm{CD}^{+}$cell counts above 200 cells per microliter, these data from HIV-infected women in the first year after delivery can help inform evolving guidelines about continuation of ARV after delivery.

Data from follow-up of the HIV-infected women enrolled to the HPTN 046 study are informative on the risk of disease progression among postpartum women in resourcelimited settings. Women with $\mathrm{CD}^{+}$lymphocyte counts between 4000 and 550 cells per microliter in pregnancy had a 1 in 3 risk of progressing to indications for ARV therapy within 1 year of delivery based on the current WHO recommendations. Given these findings, this group of women should be counseled regarding the benefits of continuing ARVs after the need for ARVs for prevention of transmission is completed. Those who are interested in continuing ARVs should be provided with support for disclosure to family members, treatment adherence, and regular follow-up visits. Pending more data on long-term outcomes from ongoing trials, women with $\mathrm{CD}^{+}$lymphocyte counts above 550 cells per microliter could be presented with information regarding the potential benefits and risks of continuing ARVs and supported in their decision to stop or continue ARVs after the indication for prevention of mother-to-child transmission of HIV has ceased.

\section{ACKNOWLEDGMENTS}

The authors thank the mothers and their children who participated in the study; the HPTN 046 study coordinators, counselors, clinicians, pharmacists, data quality and laboratory staff, and those responsible for recruitment and retention for their dedication and hard work on site; Thomas $R$. Fleming (University of Washington/Fred Hutchinson Cancer Research Center) for his contributions to study design and his strong support throughout study conduct; Scharla Estep (NIAID protocol pharmacist) for her help on pharmaceutical matters; and Avinash Shetty (Wake Forest University Health Sciences) for his contribution to study development and safety data review.

\section{REFERENCES}

1. World Health Organization. Use of antiretroviral drugs for treating pregnant women and prevention HIV infection in infants: programmatic update. 2012. Available at: http://www.who.int/hiv/pub/mtct/programmatic update2012/en/. Accessed June 7, 2013.

2. Kuhn L, Aldrovandi GM, Sinkala M, et al. Potential impact of new WHO criteria for antiretroviral treatment for prevention of mother-to-child HIV transmission. AIDS. 2010;24:1374-1377.

3. Cohen MS, Chen YQ, McCauley M, et al; for the HPTN 052 Study Team. Prevention of HV-1 infection with early antiretroviral therapy. N Engl J Med. 2011;365:493-505.

4. Nachega JB, Uthman OA, Anderson J, et al. Adherence to antiretroviral therapy during and after pregnancy in low-income, middle-income, and high-income countries: a systematic review and meta-analysis. AIDS. 2012;26:2039-2052.

5. Coovadia HM, Brown ER, Fowler MG, et al; for the HPNT046 protocol team. Efficacy and safety of an extended nevirapine regimen in infant children of breastfeeding mothers with HIV-1 infection for prevention of postnatal HIV-1 transmission (HPTN 046): a randomised, double-blind, placebo-controlled trial. Lancet. 2012;379:221-228.

6. World Health Organization. WHO case definitions of HIV for surveillance and revised clinical staging and immunological classification of HIVrelated disease in adults and children. 2007. Available at: http://www. who.int/hiv/pub/guidelines/HIVstaging150307.pdf. Accessed June 7, 2013

7. Fawzi WW, Msamanga GI, Hunter D, et al. Randomized trial of vitamin supplements in relation to transmission of HIV-1 through breastfeeding and early child mortality. AIDS. 2002;16:1935-1944.

8. Brown ER, Otieno P, Mbori-Ngacha DA, et al. Comparison of CD4 cell count, viral load, and other markers for the prediction of mortality among HIV-1-infected Kenyan pregnant women. J Infect Dis. 2009;199: 1292-1300.

9. World Health Organization. Rapid advice: antiretroviral therapy for HIV infection in adults and adolescents. 2009. Available at: http://www.who. int/hiv/pub/arv/rapid_advice_art.pdf.

10. Ekouevi D, Abrams EJ, Schlesinger M, et al; for the MTCT-Plus Initiative. Maternal CD4+ cell count decline after interruption of antiretroviral prophylaxis for the prevention of mother-to-child transmission of HIV. PLoS One. 2012;7:e43750.

11. Coria A, Noel F, Bonhomme J, et al. Consideration of postpartum management in HIV-positive Haitian women: an analysis of CD4 decline, mortality, and follow-up after delivery. J Acquir Immune Defic Syndr. 2012;61:636-643.

12. Pilotto JH, Velasque LS, Friedman RK, et al. Maternal outcomes after HAART for the prevention of mother-to-child transmission in HIVinfected women in Brazil. Antivir Ther. 2011;16:349-356. 
13. Manzi M, Zachariah R, Teck R, et al. High acceptability of voluntary counseling and HIV-testing but unacceptable loss to follow up in a prevention of mother-to-child HIV transmission programme in rural Malawi: scaling-up requires a different way of acting. Trop Med Int Health 2005; 10:1242-1250.

14. Wang B, Losina E, Stark R, et al. Loss to follow-up in a community clinic in South Africa - roles of gender, pregnancy and CD4 count. S Afr Med J. 2011;101:253-257.

15. Clouse K, Maskey M, Fox MP, et al. Delayed diagnosis of HIV and high rates of loss to follow-up among pregnant women receiving antenatal services at a primary healthcare clinic in Johannesburg, South Africa. Paper presented at: the 19th Conference on Retroviruses and Opportunistic Infections; Seattle, WA. March, 2012. Abstract 1004

16. Ahoua L, Ayikoru H, Gnauck K, et al. Evaluation of a 5-year programme to prevent mother-to-child transmission of HIV infection in Northern Uganda. J Trop Pediatr. 2010;56:43-52.
17. Ekama SO, Herbertson EC, Addeh EJ, et al. Pattern and determinants of antiretroviral drug adherence among Nigerian pregnant women. J Pregnancy. 2012;2012:851810.

18. Nachega JB, Marconi VC, van Zyl GU, et al. HIV treatment adherence, drug resistance, virologic failure: evolving concepts. Infect Disord Drug Targets. 2011;11:167-174.

19. Chilongozi D, Wang L, Brown L, et al; for the HIVNET 024 Study Team. Morbidity and mortality among a cohort of human immunodeficiency virus type 1-infected and uninfected pregnant women and their infants from Malawi, Zambia, and Tanzania. Pediatr Infect Dis J. 2008; $27: 808-814$.

20. Landes M, van Lettow M, Bedell R, et al. Mortality and health outcomes in HIV-infected and HIV-uninfected mothers at 18-20 months postpartum in Zomba district, Malawi. PLoS One. 2012;7:e44396.

21. Richardson BA, Otieno PA, Mbori-Ngacha D, et al. Hormonal contraception and HIV-1 disease progression among postpartum Kenyan women. AIDS. 2007;21:749-753. 